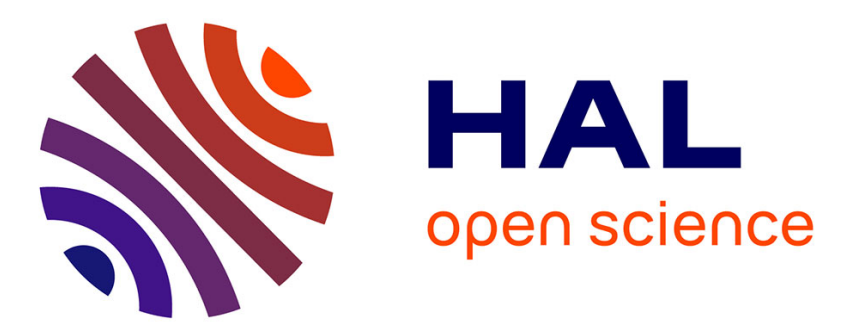

\title{
A Filtered Tabulated Chemistry Model for Large Eddy Simulation of Reactive Flows
}

Pierre Auzillon, Ronan Vicquelin, Olivier Gicquel, Nasser Darabiha, Denis

Veynante, Benoit Fiorina

\section{- To cite this version:}

Pierre Auzillon, Ronan Vicquelin, Olivier Gicquel, Nasser Darabiha, Denis Veynante, et al.. A Filtered Tabulated Chemistry Model for Large Eddy Simulation of Reactive Flows. 48th AIAA Aerospace Sciences Meeting Including the New Horizons Forum and Aerospace, Jan 2010, Orlando, United States. pp.AIAA-2010-205, 10.2514/6.2010-205 . hal-00473221

\section{HAL Id: hal-00473221 \\ https://hal.science/hal-00473221}

Submitted on 14 Apr 2010

HAL is a multi-disciplinary open access archive for the deposit and dissemination of scientific research documents, whether they are published or not. The documents may come from teaching and research institutions in France or abroad, or from public or private research centers.
L'archive ouverte pluridisciplinaire HAL, est destinée au dépôt et à la diffusion de documents scientifiques de niveau recherche, publiés ou non, émanant des établissements d'enseignement et de recherche français ou étrangers, des laboratoires publics ou privés. 


\title{
A filtered tabulated chemistry model for Large Eddy Simulation of reactive flows
}

\author{
P. Auzillon ${ }^{* a}$, R. Vicquelin ${ }^{a, b}$, O. Gicquel ${ }^{a}$, \\ N. Darabiha ${ }^{a}$, D. Veynante ${ }^{a}$ and B. Fiorina ${ }^{a}$ \\ ${ }^{a}$ EM2C - CNRS, UPR 288, Ecole Centrale Paris \\ 92295 Châtenay Malabry, France
}

${ }^{b}$ GDF SUEZ, Pôle CHENE, Centre de Recherche et d'Innovation Gaz et Energies Nouvelles

93211 Saint-Denis la Plaine, France

\begin{abstract}
A new model called F-TACLES (Filtered Tabulated Chemistry for LES) is developed to introduce tabulated chemistry methods in LES of turbulent premixed combustion. The main objective is to recover the correct propagation speed of the filtered flame front when the sub-grid scale wrinkling vanishes. The filtered flame structure is mapped by 1-D filtered laminar premixed flames. The methodology is first applied to 1-D filtered laminar flames. Computations show the capability of the model to recover the laminar flame speed and the correct chemical structure when flame wrinkling is fully resolved on the LES filter scale. The model is then extended to turbulent regimes by introducing sub-grid scale wrinkling effects on the flame propagation. LES's of a 3-D turbulent premixed flame are performed on different grids, with different flame filter sizes. Objectives are to analyze the influence of the grid size, the flame filter size and the sub-grid flame wrinkling on the model performances. All these computations are compared to experimental data.
\end{abstract}

\section{Introduction}

Objectives of Large Eddy Simulations of combustion are to predict unsteady features such as flame ignition, extinction or pollutant emissions. These phenomena are driven by chemical kinetics and require therefore an accurate description of combustion chemistry. Hydrocarbon combustion generally involves hundreds of species. In complex chemistry simulations, one balance equation must be added to the Navier-Stokes equation for each species present in the detailed mechanism. Therefore, despite the continual increase of computational power, simulations of practical combustion systems under complex chemistry assumption remains impossible.

Various technique exist to tabulate the chemistry. Some are based on mathematical analysis, ${ }^{1}$ and other are based on physical consideration. ${ }^{2-4}$ In practical reactive systems, the chemical composition may be considered evolving on a reduced subspace ${ }^{1}$ called manifold. Various techniques such as ILDM ${ }^{1}$ (Intrinsic Low Dimensional Manyfold), FPI $^{2,3}$ (Flame Prolongation of ILDM) or FGM 4 (Flamelet Generated Manifold) have been proposed to calculate these manifolds. Thermochemical quantities such as species mass fractions and reaction rates are then tabulated as a function of a reduced set of variables describing the manifolds. Balance equations of these variables are then solved instead of the direct solving of the entire set of species equations. These equations are coupled with the Navier-Stokes equations to describe the reactive flow.

A recurrent problem that occurs in coupling tabulated chemistry with Large Eddy Simulation is the numerical resolution of the filtered flame front. Indeed in practical LES meshes, the flame thickness is typically thinner than the grid size. As the progress variable source term is very stiff, the flame front structure cannot be directly resolved on practical LES grids, leading to numerical issues. To overcome this difficulty, dedicated models have been developed under simplified chemistry assumptions. The main

*Corresponding author : pierre.auzillon@centraliens.net 
commonly used strategies are to artificially thicken the flame, ${ }^{5,6}$ or track the inner layer using level set techniques $^{7}$ or thicken by filtering the reaction rate at scales larger than the grid size.

In order to introduce tabulated chemistry in LES, a new strategy has recently been introduced by Fiorina et al. ${ }^{8}$ The model called F-TACLES (Filtered tabulated chemistry for LES) consists in coupling filtered flow governing equations with a filtered chemical database. Filtered reaction rates, filtered diffusion terms and filtered diffusion fluxes are carefully modeled. The formulation insures a proper description of the flame front propagation over the entire domain of the LES diagram.

For numerical reasons, the filter size $\Delta$ has to be larger than the mesh size, $\Delta_{x}$. Indeed a sufficient number of grid points across the sources term is required to resolve the flame front. As the mesh size, $\Delta_{x}$ is imposed by the cpu time and available memory, the effect on the front flame propagation of the ratios $\Delta / \Delta_{x}$ and $\Delta / \delta_{l}$, where $\delta_{l}$ is the laminar flame thickness, must be understood. For this purpose, parametric studies are performed on 1-D filtered flames. In this article after a brief description of the F-TACLES model, the impact of the ratio $\Delta / \Delta_{x}$ on the filtered flame propagation is investigated on 1-D premixed flame configurations. Then, studies of the effect of $\Delta / \delta_{l}$ on multi-dimensional flows are investigated by simulations of a turbulent premixed burner computed with two different grids and filter sizes.

\section{The F-TACLES model}

\section{A. Coupling tabulated chemistry with LES}

In premixed combustion, chemistry trajectories can be estimated from 1-D laminar premixed flames computed with detailed chemistry. ${ }^{2}$ For a given set of values of initial pressure and temperature and equivalence ratio, thermodynamical and chemical quantities are tabulated as functions of one unique progress variable, $c$, related to temperature or to a combination of species mass fractions. $c$ is normalized such that, $c=0$ corresponds to fresh gases and $c=1$ to burnt gases. The chemical database parametrized by $c$ is then coupled to the flow field by the addition of the progress variable balance equation to the Navier-Stokes equations.

In LES, flow and progress variable equations should be filtered. Under unity Lewis number assumption, the filtered system of equations reads :

$$
\begin{aligned}
\frac{\partial \bar{\rho}}{\partial t}+\nabla \cdot(\bar{\rho} \widetilde{\mathbf{u}})= & 0 \\
\frac{\partial \bar{\rho} \widetilde{\mathbf{u}}}{\partial t}+\nabla \cdot(\bar{\rho} \widetilde{\mathbf{u}} \widetilde{\mathbf{u}})= & -\nabla \cdot(\bar{P} \underline{\delta})+\nabla \cdot \bar{\tau}-\nabla \cdot(\bar{\rho} \widetilde{\mathbf{u}}-\bar{\rho} \widetilde{\mathbf{u}}) \\
\frac{\partial \bar{\rho} \widetilde{c}}{\partial t}+\nabla \cdot(\bar{\rho} \widetilde{\mathbf{u}} \widetilde{c}= & \nabla \cdot(\overline{\rho D \nabla c})-\nabla \cdot(\bar{\rho} \widetilde{\mathbf{u} c}-\bar{\rho} \widetilde{\mathbf{u}} \widetilde{c})+\overline{\dot{\omega}}_{c} \\
\frac{\partial \bar{\rho} \widetilde{E}}{\partial t}+\nabla \cdot(\bar{\rho} \widetilde{\mathbf{u}} \widetilde{E})= & -\nabla \cdot(\overline{P \mathbf{u}} \underline{\delta})+\nabla \cdot(\overline{\tau \mathbf{u}})-\nabla \cdot(\bar{\rho} \widetilde{\mathbf{u} E}-\bar{\rho} \widetilde{\mathbf{u}} \widetilde{E}) \\
& +\nabla \cdot(\overline{\rho D \nabla h})+\overline{\dot{\omega}}_{E} \\
\bar{P}= & \bar{\rho} \frac{R}{W} \widetilde{T}
\end{aligned}
$$

where $\rho$ is the density, $\mathbf{u}$ the velocity vector, $P$ the pressure, $\underline{\delta}$ the unit tensor, $\tau$ the laminar viscous tensor, $E=H-P / \rho$ with $H$ the total enthalpy, $h$ the sensible enthalpy, $D$ is the diffusivity, $R$ the ideal gas constant and $W$ the mean molecular weight. The overbar denotes the spatial filtering operation,

$$
\bar{\phi}(x)=\int_{-\infty}^{+\infty} F\left(x-x^{\prime}\right) \phi\left(x^{\prime}\right) d x^{\prime},
$$

where $\phi$ represents reactive flow variables and velocity components. The tilde operator denotes the density-weighted filtering defined by $\bar{\rho} \widetilde{\phi}=\overline{\rho \phi}$.

In the previous equations, $(2-4)$, the unresolved transport terms, $\nabla \cdot(\bar{\rho} \widetilde{\mathbf{u}} \phi-\bar{\rho} \widetilde{\mathbf{u}} \bar{\phi})$, the filtered laminar diffusion terms $\overline{\rho D \nabla \phi}$ and the filtered source terms, require closure models. The model constraints are both to ensure a correct flame propagation and to recover the chemical structure of the filtered flame under two possible situations : (1) the flame wrinkling is fully resolved at the LES filter size, and (2) wrinkling occurs at the sub-grid scale and affects the filtered flame speed. 


\section{B. Model formulation}

The flame structure in the direction $\mathbf{n}$ normal to the flame front is assumed identical to the structure of a 1-D freely propagating laminar premixed planar flame obtained by using detailed chemical mechanism involving $N_{s}$ species. From this reference flame structure and using the filter operators, the a priori filtered flame structure is determined. For instance, for a given filter size $\Delta$, any filtered fluxes or filtered thermochemical quantities of a filtered laminar flame planar are determined using the gaussian filter :

$$
F(\mathbf{x})=\left(\frac{6}{\pi \Delta^{2}}\right)^{1 / 2} \exp \left(-\frac{6 \mathbf{x}^{2}}{\Delta^{2}}\right)
$$

In the following, for any variable $\phi$, by the notation $\phi[\widetilde{c}, \Delta]$ we mean that $\phi$ is tabulated in a 2-D look-up table with coordinates $\widetilde{c}$ and $\Delta$.

\section{Filtered chemical reaction source terms}

The filtered source terms for the progress variable and the energy equations are directly determined from the look-up database shown in Fig. 1 :

$$
\widetilde{\dot{\omega}}_{\varphi}=\widetilde{\dot{\omega}}_{\varphi}^{*}[\widetilde{c}, \Delta] .
$$

where, $\varphi$ denotes $c$ or $E$ quantities and the ${ }^{*}$ superscript denotes quantities extracted from a 1-D unstretched laminar premixed flame.

\section{Filtered laminar diffusion terms}

These terms are usually approximated as:

$$
\nabla \cdot(\overline{\rho D \nabla \varphi}) \approx \nabla \cdot(\bar{\rho} D \nabla \widetilde{\varphi}) .
$$

However, as shown in ${ }^{8}$ this approximation introduces large errors. In the present work, the filtered diffusion term for eq. 3 is modeled by:

$$
\begin{aligned}
\nabla \cdot(\overline{\rho D \nabla c}) & \approx-\nabla \cdot(\overline{\rho D|\nabla c|} \mathbf{n}) \\
& =-\nabla \cdot\left(\overline{\rho D\left|\frac{\partial c^{*}}{\partial x^{*}}\right|} \mathbf{n}\right) \\
& =\nabla \cdot\left(\alpha_{c}[\widetilde{c}, \Delta] \bar{\rho} D \nabla \widetilde{c}\right),
\end{aligned}
$$

where the vector normal to the flame front, $\mathbf{n}=-\nabla c /|\nabla c|$, points towards the fresh reactants. A correction factor $\alpha_{c}(\widetilde{c})$ is introduced:

$$
\alpha_{c}[\widetilde{c}, \Delta]=\frac{\overline{\rho D\left|\frac{\partial c^{*}}{\partial x^{*}}\right|}}{\bar{\rho} D\left|\frac{\partial \widetilde{c^{*}}}{\partial x^{*}}\right|}
$$

The quantity $\alpha_{c}[\widetilde{c}, \Delta]$ is estimated from the 1-D filtered flame solution and is tabulated as a function of $\widetilde{c}$ for a given value of the filter size $\Delta$ as shown in Fig. 2 .

Similarly, the filtered laminar energy diffusion term is written as:

$$
\nabla \cdot(\overline{\rho D \nabla h})=\nabla \cdot\left(\alpha_{E}([\widetilde{c}, \Delta]) \bar{\rho} D \nabla \widetilde{h}\right) .
$$

where the correction factor $\alpha_{E}[\widetilde{c}, \Delta]$ is defined as:

$$
\alpha_{E}[\widetilde{c}, \Delta]=\frac{\overline{\rho D\left|\frac{\partial h^{*}}{\partial x^{*}}\right|}}{\bar{\rho} D\left|\frac{\partial h^{*}}{\partial x^{*}}\right|} .
$$




\section{Sub-grid scale convection terms}

The displacement speed $\mathbf{s}_{\mathbf{d}}$, measuring the fame front local speed relative to the flow, i.e. the difference between the absolute flow velocity $\mathbf{u}$ and the absolute fame front speed $\mathbf{w}$, is first introduced:

$$
\mathbf{u}=\mathbf{w}+\mathbf{s}_{\mathbf{d}}
$$

The filtered flame front speed $\mathbf{w}$ should remain constant across the flame brush $(\widetilde{\mathbf{w}}=\overline{\mathbf{w}}=\mathbf{w})$, therefore after replacing the flow velocity by relation 16 , the sub-grid scale convection term then reads:

$$
-\nabla \cdot(\bar{\rho} \widetilde{\mathbf{u} \varphi}-\bar{\rho} \widetilde{\mathbf{u}} \widetilde{\varphi})=-\nabla \cdot\left(\widetilde{\rho} \widetilde{\mathbf{s}_{\mathbf{d}} \varphi}-\bar{\rho} \widetilde{\mathbf{s}_{\mathbf{d}}} \widetilde{\varphi}\right)
$$

In a 1-D laminar premixed flame the laminar flame speed $S_{l}^{0}$ and the fresh gas mixture density $\rho_{0}$ are related to the displacement speed through the following relation:

$$
\rho_{0} S_{l}^{0}=\bar{\rho} \widetilde{s_{d}^{*}}
$$

Therefore, under the assumption that the flame remains planar at the sub-grid scale level, the unresolved convection terms are directly estimated from the reference 1-D laminar premixed flame:

$$
\begin{aligned}
-\nabla \cdot(\bar{\rho} \widetilde{\mathbf{u} \varphi}-\bar{\rho} \widetilde{\mathbf{u}} \widetilde{\varphi}) & =-\frac{\partial}{\partial x^{*}}\left(\bar{\rho} \widetilde{s_{d}^{*} \varphi^{*}}-\bar{\rho} \widetilde{s}_{d}^{*} \widetilde{\varphi}^{*}\right) \\
& =-\rho_{0} S_{l}^{0}\left(\frac{\partial \overline{\varphi^{*}}}{\partial x^{*}}-\frac{\partial \widetilde{\varphi^{*}}}{\partial x^{*}}\right) . \\
& =\Omega_{\varphi}[\widetilde{c}, \Delta]
\end{aligned}
$$

where the quantity $\Omega_{\varphi}[\widetilde{c}, \Delta]$ is also estimated from the 1-D filtered flame solution and is tabulated as a function of $\widetilde{c}$ for a filter size $\Delta$ as shown in Fig. 1 .

\section{Pressure term}

In a similar way, the pressure term in the energy equation (Eq. 4) is written as:

$$
\begin{aligned}
-\nabla \cdot(\overline{P \overline{\mathbf{u}}} \underline{\delta}) & =-\nabla \cdot(\bar{P} \widetilde{\mathbf{u}} \underline{\delta})-(\nabla \cdot(\overline{P \overline{\mathbf{u}}} \underline{\delta})-\nabla \cdot(\bar{P} \widetilde{\mathbf{u}} \underline{\delta})) \\
& =-\nabla \cdot(\bar{P} \widetilde{\mathbf{u}} \underline{\delta})-(\nabla \cdot(\bar{\rho} \widetilde{r T \mathbf{u}} \underline{\delta})-\nabla \cdot(\bar{\rho} \widetilde{r T} \widetilde{\mathbf{u}} \underline{\delta})) \\
& \approx-\nabla \cdot(\bar{P} \widetilde{\mathbf{u}} \underline{\delta})+\Omega_{p}[\widetilde{c}, \Delta]
\end{aligned}
$$

with

$$
\Omega_{p}[\widetilde{c}, \Delta]=-\rho_{0} S_{l}^{0}\left(\frac{\partial\left(\overline{r T^{*}}\right)}{\partial x^{*}}-\frac{\partial\left(\widetilde{r T^{*}}\right)}{\partial x^{*}}\right)
$$

\section{Summary of F-TACLES equations}

As the turbulence induces flame wrinkling, the flame surface area at sub-grid scale increases. As a consequence, the filtered flame front takes a new velocity : the turbulent flame speed, $S_{t}$. This turbulent flame speed is related to the laminar flame speed by the wrinkling factor $\Xi .{ }^{9}$ The model is derived to ensure that the filtered flame front propagates at the turbulent flame speed. The filtered flame thickness is assumed to be only related to the filter size $\Delta$ and is not altered by small-scale eddies. Then, the filtered progress variable turbulent reaction rate is modeled by:

$$
\overline{\dot{\omega}}_{c_{t}}=\Xi \cdot \overline{\dot{\omega}}_{c}^{*}[\widetilde{c}, \Delta]
$$

and the turbulent diffusion term is expressed as follows:

$$
\Omega_{c_{t}}=-(\nabla \cdot(\bar{\rho} \widetilde{\mathbf{u} c}-\bar{\rho} \widetilde{\mathbf{u}} \widetilde{c}))_{t}=\Xi \Omega_{c}[\widetilde{c}, \Delta]+(\Xi-1) \nabla \cdot\left(\alpha_{c}[\widetilde{c}, \Delta] \bar{\rho} D \nabla \widetilde{c}\right)
$$

The first term on the r.h.s corresponds to the thermal expansion and the second one models the unresolved turbulent fluxes. This formulation ensures that the filtered flame front propagates at the velocity $S_{t}$ in the normal direction because every r.h.s. term has been multiplied by the wrinkling factor $\Xi$. The same procedure is applied to the energy equation. 


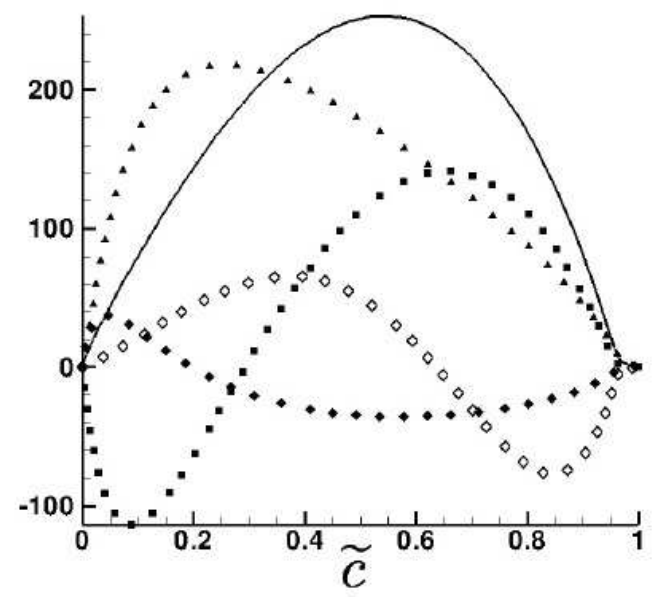

Figure 1. Budget terms (in $\mathrm{kg} \cdot \mathrm{m}^{-3} \cdot \mathrm{s}^{-1}$ ) of the filtered progress variable balance equation of a filtered 1-D planar methane/air premixed laminar planar flame as a function of $\widetilde{c}$ for $\Delta=25 \delta_{l}: \quad-: \nabla \cdot(\bar{\rho} \widetilde{\mathbf{u}} \widetilde{c}), \diamond:$ $\nabla\left(\overline{\rho D \frac{\partial c^{*}}{\partial x^{*}}}\right), \mathbf{\square}: \Omega_{c}, \boldsymbol{\Delta}: \overline{\dot{\omega}}_{c}, \diamond: \nabla\left(\overline{\rho D} \frac{\partial \widetilde{c}^{*}}{\partial x^{*}}\right)$.

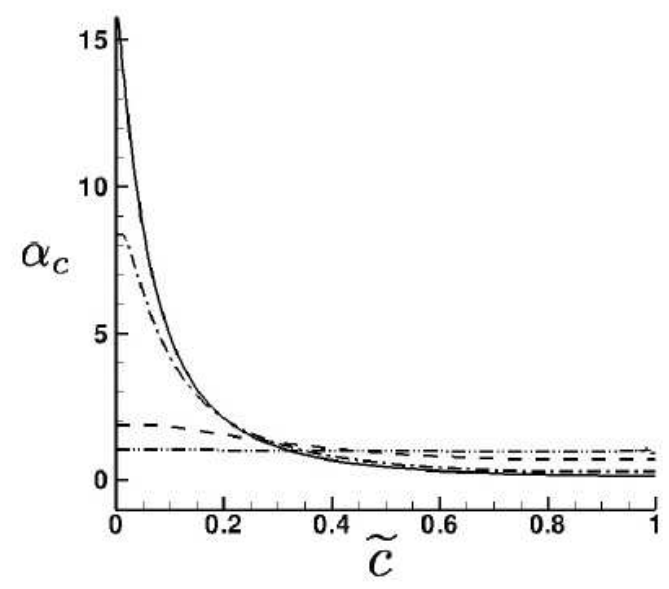

Figure 2. Diffusion correction factor $\alpha_{c}$ as a function of $\widetilde{c}$ for different values of $\Delta$. Dashed dotted-dotted lines: $\Delta=0.2 \delta_{l}$, Dashed lines: $\Delta=1 \delta_{l}$, Dashed dotted lines: $\Delta=5 \delta_{l}$, Solid lines: $\Delta=25 \delta_{l}$. 
In this case, the equations of the F-TACLES model can be written as follows (a comparison with the laminar model is summarized in Table 1 ) :

$$
\begin{aligned}
\frac{\partial \bar{\rho} \tilde{\mathbf{u}}}{\partial t}+\nabla \cdot(\bar{\rho} \widetilde{\mathbf{u}})= & -\nabla \bar{P}+\nabla \cdot \widetilde{\tau}+\nabla \cdot \bar{\tau}^{t} \\
\frac{\partial \bar{\rho} \widetilde{c}}{\partial t}+\nabla \cdot(\bar{\rho} \widetilde{\mathbf{u}} \widetilde{c})= & \Xi \nabla \cdot\left(\alpha_{c}[\widetilde{c}, \Delta] \bar{\rho} D \nabla \widetilde{c}\right)+\Xi \Omega_{c}[\widetilde{c}, \Delta]+\Xi \overline{\dot{\omega}}_{c}[\widetilde{c}, \Delta] \\
\frac{\partial \bar{\rho} \widetilde{E}}{\partial t}+\nabla \cdot(\bar{\rho} \widetilde{\mathbf{u}} \widetilde{E})= & -\nabla \cdot(\bar{P} \widetilde{u} \underline{\delta})+\Xi \Omega_{p}[\widetilde{c}, \Delta]+\nabla \cdot(\widetilde{\tau} \widetilde{\mathbf{u}})+\Xi \Omega_{E}[\widetilde{c}, \Delta] \\
& +\Xi \nabla \cdot\left(\alpha_{E}[\widetilde{c}, \Delta] \bar{\rho} D \nabla \widetilde{h}\right)+\Xi \overline{\dot{\omega}}_{E}[\widetilde{c}, \Delta] .
\end{aligned}
$$

Note that the sub-grid scale turbulent fluxes $\nabla \cdot \bar{\tau}^{t}$ are modeled using the Smagorinsky model. Different alternatives exist to estimate the sub-grid flame wrinkling that appears in Eqs. 29 and 30. It can either be estimated from analytical models ${ }^{9-11}$ or from the solution of a flame surface density balance equation. ${ }^{12,13}$

\section{Numerical simulations with F-TACLES}

In the present method, the filtered size $\Delta$ is a crucial parameter. Therefore, different computations are performed in order to investigate the impact of the ratios $\Delta / \Delta_{x}$ and $\Delta / \delta_{l}$. Several1-D flames and a complex 3-D swirler chamber are computed. The first ones show the ability of the F-TACLES model to predict the flame speed when the wrinkling is fully resolved in the LES. The 3-D case quantifies the impact of the ratio $\Delta / \delta_{l}$ when the flame is wrinkled at the sub-grid scale.

\section{A. Results on 1-D Flame}

In order to quantify the ability of F-TACLES method to reproduce the correct flame front propagation speed with different ratios $\Delta / \Delta_{x}$ and $\Delta / \delta_{l}$, a set of filtered steady 1-D laminar premixed flames is computed. Stoichiometric methane/air combustion is considered taking into account detailed chemistry effects. The PREMIX ${ }^{14}$ solver is used with a modified version of the GRI 3.0 mechanism (http://www.berkeley.edu/gri_mech/) involving $N_{s}=70$ species and 463 elementary reactions. The laminar flame thickness $\delta_{l}$ is equal to $0.637 \mathrm{~mm}$ and the laminar flame speed, $S_{l}^{0}$, is $0.39 \mathrm{m.s}{ }^{-1}$. The filter operator given by Eq. (7) is then applied to the 1-D laminar flame solution. Each simulation is, then, performed on uniform meshes. In this configuration, there is no sub-grid scale wrinkling. Different filter sizes are tested. For each filter size, the reference solution is obtained by filtering the 1-D laminar premixed flame detailed chemistry solution. Each simulation is initialized with this reference and ends at physical time defined by $t_{\text {end }}=50 \Delta / S_{l}^{0}$.

A comparison between the numerical solutions on uniform mesh (solid lines) and the reference solution

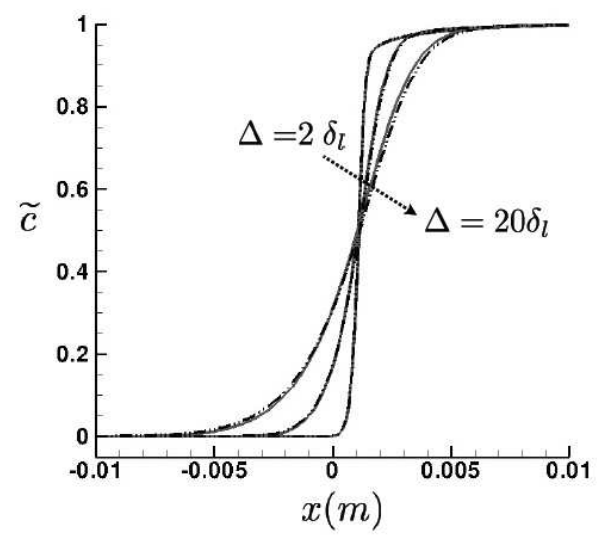

Figure 3. Filtered 1-D premixed flame solutions. Filtered progress variable (solid) compared to the reference solution (dashed) for $\Delta / \delta_{l}=2,10$ and 20 .

(dashed line) is first shown in Fig. 3 for different values of $\Delta / \delta_{l}$. The predicted filtered progress variable profiles match the reference solution for all the filter size values. Fig. 4(a) shows that the predicted filtered front propagation speed $S_{\Delta}$ (square symbols) remains very close to the expected laminar flame 
speed for various values of $\Delta / \delta_{l}$. However as shown in Fig. 4(b), in order to correctly predict the laminar flame speed, the mesh resolution has to verify the condition $\delta_{\widetilde{c}} / \Delta_{x} \geq 5$, where $\delta_{\widetilde{c}}=1 / \max \left(\left|\frac{\partial \widetilde{c}}{\partial x}\right|\right)$ is an estimation of the filtered flame thickness. Below this critical value, the number of grid points in the flame front is not sufficient, the filter flame front propagation speed is overestimated and does not reproduce the laminar flame speed.

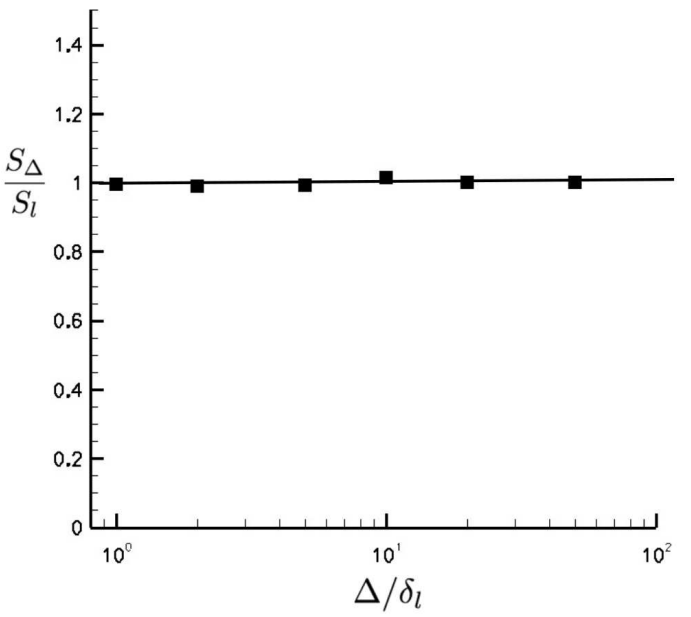

(a) $\Delta / \Delta_{x}=50$

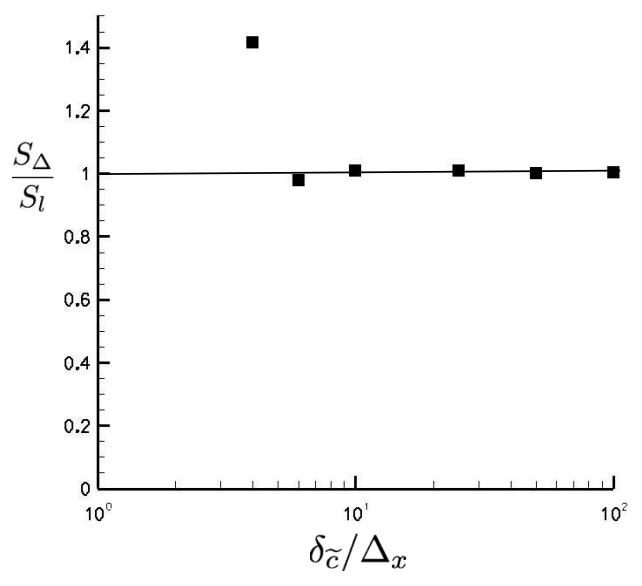

(b) $\Delta / \delta_{l}=20$

Figure 4. Predicted flame speed as a function of $\Delta / \delta_{l}$ (left) and $\Delta / \Delta_{x}$ (right). $\delta_{\widetilde{c}}$ is the filter flame thickness

\section{B. Large Eddy Simulation of the PRECCINSTA configuration}

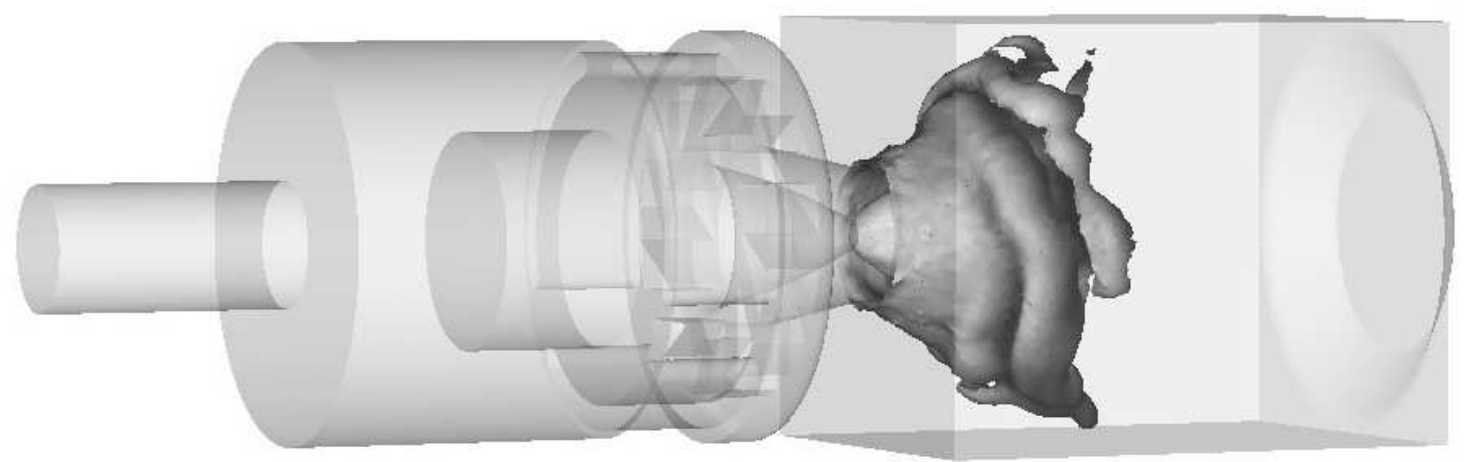

Figure 5. Iso-surface of the temperature $(T=1600 \mathrm{~K})$ and view of the swirler PRECCINSTA

The F-TACLES method is applied to study a 3-D complex configuration, PRECCINSTA, experimentally investigated by Meier et al. ${ }^{15}$ The geometry is representative of the combustion chamber of an gas turbine (Fig. 5). It consists of a plenum, a swirl-injector and a combustion chamber. We assume that the mixing is fast leading to a perfectly premixed regime. A methane/air mixture is injected into the plenum with an equivalence ratio of 0.83 , the inlet condition chosen in this simulation corresponds to an air mass rate of $12.3 \mathrm{~g} / \mathrm{s}$ and a methane air flow rate of $0.6 \mathrm{~g} / \mathrm{s}$.

For the simulation, the first step is to compute a 1-D methane/air flame with PREMIX ${ }^{14}$ using the GRI 3.0 mechanism (http://www.berkeley.edu/gri_mech/) at an equivalence ratio of $\phi=0.83$. Then, this flame solution is filtered by a Gaussian function with a filter width of $\Delta$. This filter size ensure a sufficient number of grid points in the filtered flame front. The tests on 1-D flame have shown that the F-TACLES model reproduces the flame speed with a good agreement (Fig. 4(b)) for 5 cells in the filtered flame front, $\delta_{\widetilde{c}} / \Delta_{x} \geq 5$. In this $3 \mathrm{D}$ calculation, $\delta_{\widetilde{c}} / \Delta_{x}$ is set 6 for all computations. The progress variable is defined by $\mathrm{c}=\bar{Y}_{\mathrm{CO}_{2}} / Y_{\mathrm{CO}_{2}}^{e q}$. All the filtered quantities required by the models are tabulated as a function of $\tilde{c}$ for the filter size considered.

The F-TACLES model has been implemented into the compressible LES code AVBP. ${ }^{16}$ The third order finite element scheme TTGC $^{17}$ and a third order Runge Kutta explicit time step are used. The 
sub-grid flame wrinkling is estimated from the analytical model developed by Colin et al. ${ }^{9}$ Two different meshes are used to study the influence of the sub-grid wrinkling, With a fine mesh, as a larger contribution of the total flame surface is resolved, the wrinkling is less important than in the case of a coarse mesh (the maximum value decreases from 6.9 to 4.3). The fine unstructured mesh used to perform the computation contains 12.7 millions elements which are almost uniform in flame area. The second mesh has the same type of distribution but contains only 3 millions cells. A filter length $\Delta$ of $\Delta=20 \delta_{l}$ is used for the computation on the fine mesh.For the second mesh, $\Delta$ is set to $\Delta=40 \delta_{l}$. Mean and resolved Root Mean Square (RMS) quantities are computed by time averaging LES solutions over a physical time that corresponds to 6 flow-through times based on the fresh gas inlet velocity. Mean temperature, temperature

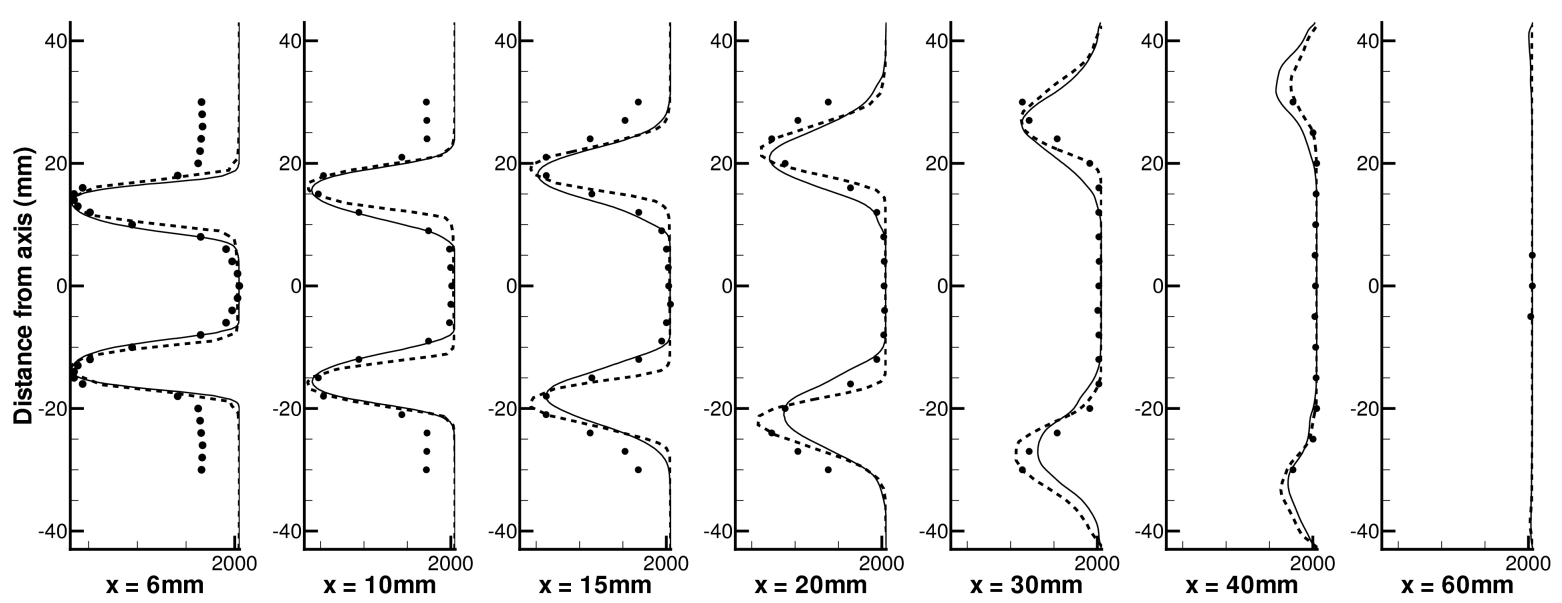

Figure 6. Mean values of temperature, case $\phi=0.83$. $\bullet$ : measurements. - : simulation with $\Delta / \delta_{l}=20$. --: simulation with $\Delta / \delta_{l}=40 \cdot \mathbf{x}=\mathbf{0}$ matches the swirler exit

RMS, $\mathrm{CO}_{2}$ mass fractions and $\mathrm{CO}_{2}$ mass fractions RMS are plotted on Figs. 6 through 9, respectively. A very good agreement is observed between experimental and numerical profiles, which demonstrates that the correct flame angle and mean flame thickness are reproduced by the model for the two filter sizes. Because heat losses have not been considered when generating the chemical database and in the numerical simulation, the LES slightly over-estimates the temperature profiles close to the combustion chamber wall, in the outer recirculation zone for $x<20 \mathrm{~mm}$ and at radial distances larger than $20 \mathrm{~mm}$ from the jet axis. Note that heat losses effects on the flame structure can be taken into account with the addition of the enthalpy as a second coordinate of the chemistry look-up table. ${ }^{3,18}$

Figures. 7 and 9 show a comparison between resolved LES RMS and measured RMS of the temperature and the $\mathrm{CO}_{2}$ mass fraction, respectively. As the plotted LES RMS does not include the sub-grid scale RMS, conclusions regarding the model performance in terms of flame turbulence interactions are more difficult. However, it is observed that LES RMS remains lower than measured RMS for each filter size, as expected from theory. In addition, we observed an increase of the RMS values with the fine mesh. As the flame and the turbulence are resolved with more resolution, the fluctuations increased.

Finally, Fig. 10 indicates the flame position in the Pitsch LES regime diagram ${ }^{19}$ for turbulent premixed combustion, where the ratio $\Delta / \delta_{l}$ is expressed as a function of the Karlovitz number $K a$ in logarithmic scale. In LES, the Karlovitz number is related to the sub-grid velocity fluctuations $v_{\Delta}^{\prime}$ and laminar flame scales ${ }^{19}$ :

$$
K a^{2}=\frac{\delta_{l}}{S_{l}^{0^{3}}} \varepsilon=\frac{v_{\Delta}^{\prime}}{S_{l}^{0}} \frac{\delta_{l}}{\Delta}
$$

where $\varepsilon$ is the kinetic energy transfer rate. The sub-grid velocity fluctuations are computed as follows :

$$
v_{\Delta}^{\prime}=\frac{\mu_{t}}{\bar{\rho} C_{k} \Delta \sqrt{3 / 2}}
$$

where the turbulent viscosity $\mu_{t}$ is estimated from Smagorinsky model. In this diagram $K a<1$, combustion takes place in the corrugated flame regime while the thin reaction zone regime is observed when $K a>1$. Computational nodes located in the filtered flame front are considered, i.e. for $0.01<\widetilde{c}<0.99$, and are plotted in the Pitch diagram (horizontal thick solid black lines in Fig. 10). In our simulations, 

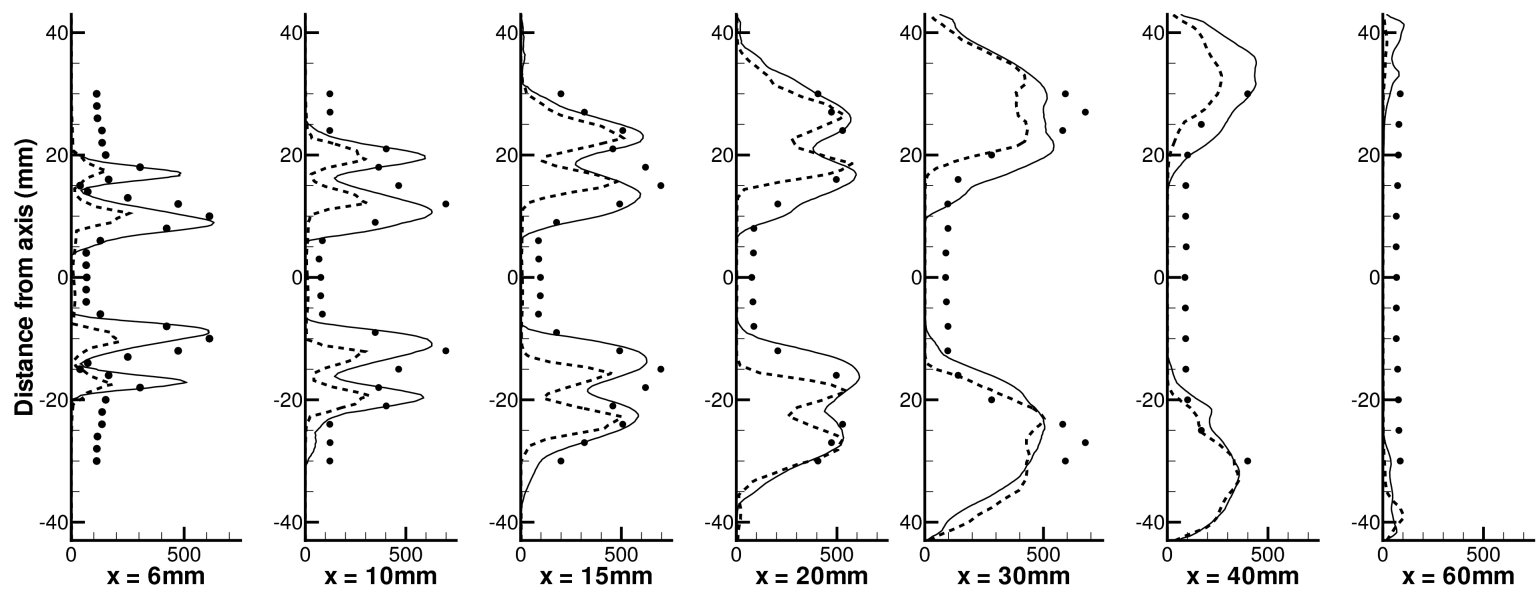

Figure 7. RMS values of temperature, case $\phi=0.83$. $\bullet$ measurements. - : simulation with $\Delta / \delta_{l}=20$. -- : simulation with $\Delta / \delta_{l}=40 \cdot \mathrm{x}=0$ matches the swirler exit

as only two values of $\Delta$ are considered (one for each mesh), the scatter plot is reduced to two lines : one for $\Delta / \delta_{l}=20$ and the other for $\Delta / \delta_{l}=40$. The smallest size of the flame wrinkling is given by the Gibson length ${ }^{20}$ :

$$
\frac{\Delta}{l_{G}}=\frac{v_{\Delta}^{\prime}}{S_{l}^{0}}
$$

The substitution of Eq. 33 into Eq. 31 shows that $\Delta=l_{G}$ condition corresponds to $\Delta / \delta_{l}=K a^{-2}$ represented by a line of slope -2 in the Pitch diagram (Fig. 10). In the corrugated flame regime, when the Gibson length becomes larger than the filter width, the sub-grid velocity fluctuation $v_{\Delta}^{\prime}$ is smaller than the laminar flame speed $S_{l}^{0}$. In such cases, there are enough points in the flame front and the flame wrinkling is fully resolved at the LES filter scale. At the opposite, on the right side of the $l_{G}=\Delta$ line, sub-grid scale wrinkling is more important and will impact the filtered flame front propagation speed $S_{\Delta}$. On Fig. 10 the horizontal lines corresponding to nodes located in the flame front do not show what percentage of these points are located in the zone with a wrinkling at the sub-grid scale and for what percentage of them the wrinkling is fully resolved. This can be displayed by plotting the number of points in the flame front $(0.01<\widetilde{c}<0.99)$ as a function of the Karlovitz number (Fig. 11). For each simulation, it can be observed that most of the points are located in the corrugated flame regime $(K a<1)$. The chemical flame structure remains therefore laminar as assumed in the present model. Also, for a substantial area of the flame surface ( about $30 \%$ for the fine mesh), the Gibson length $l_{G}$ is larger than the filter width and consequently the flame wrinkling is fully resolved. However, for the coarse mesh with $\Delta / \delta_{l}=40$, only $5 \%$ of the surface area are under the Gibson limit. The comparison with the fine grid shows that as meshes will be finer in the future with the increase of computational power, this trend should be emphasized. This shows that ensuring a proper propagation of the laminar flame front when deriving a turbulent combustion model is a crucial point in modeling.

\section{Conclusion}

A new model called F-TACLES (Filtered Tabulated Chemistry for LES) is developed to introduce tabulated chemistry methods in LES of premixed combustion. A filtered 1-D laminar premixed flame is used to build a filtered chemical look-up table. The model performances are demonstrated on 1-D filtered laminar flame computations. As long as the ratio $\delta_{\widetilde{c}} / \Delta_{x}$ is larger than 5 , the F-TACLES reproduces the actual laminar flame speed for various filter sizes. Finally the proposed strategy has been applied to perform 3-D computations of a swirled turbulent premixed flame in order to study the influences of filter size on the results, highlighting the importance of the fully resolved wrinkling behavior of the combustion model. 

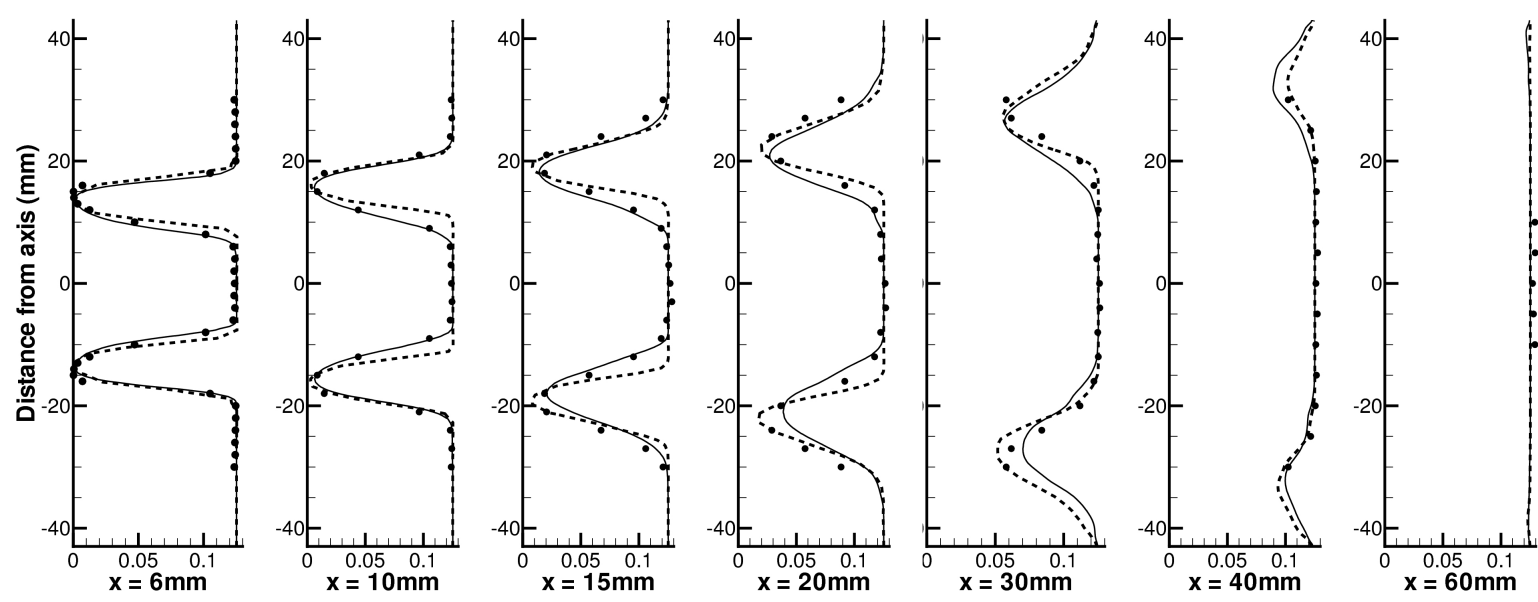

Figure 8. Mean values of $\mathrm{CO}_{2}$ mass fraction, case $\phi=0.83$. $\bullet$ : measurements. - : simulation with $\Delta / \delta_{l}=20$. - - : simulation with $\Delta / \delta_{l}=40 \cdot \mathbf{x}=\mathbf{0}$ matches the swirler exit
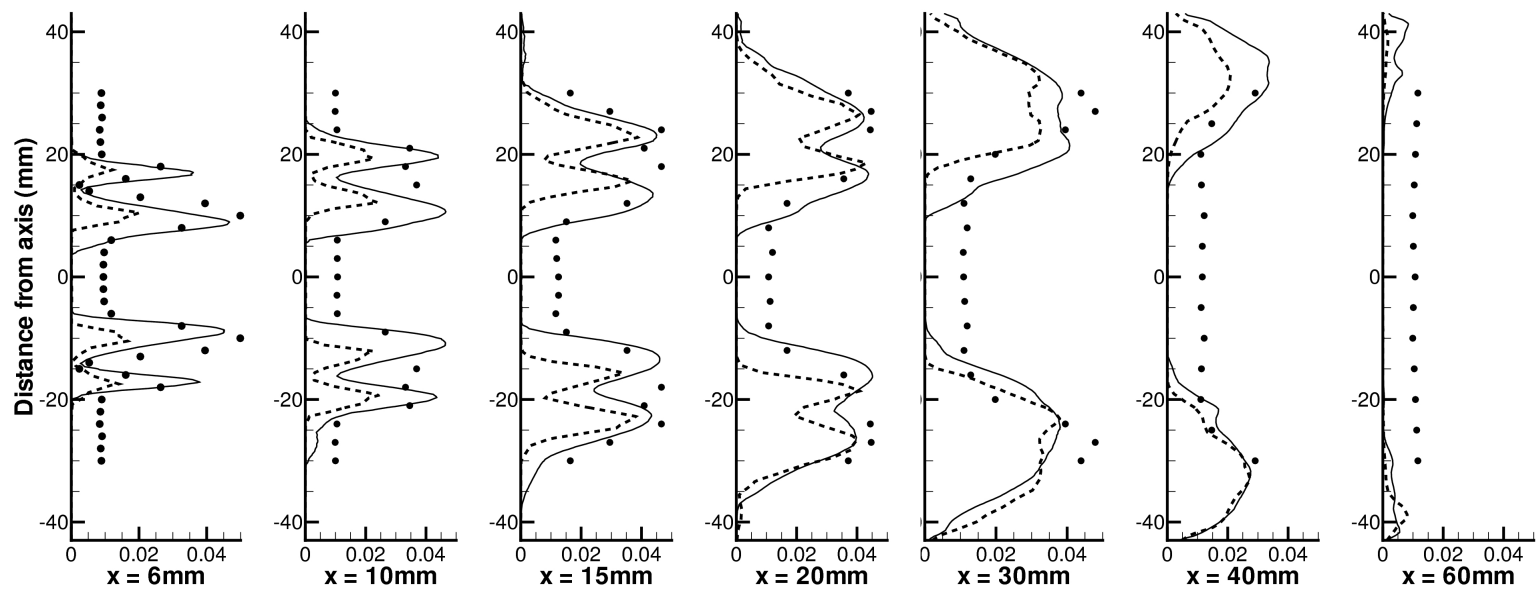

Figure 9. RMS values of $\mathrm{CO}_{2}$ mass fraction, case $\phi=0.83$. •: measurements. - : simulation with $\Delta / \delta_{l}=20$. --: simulation with $\Delta / \delta_{l}=40 . \mathbf{x}=\mathbf{0}$ matches the swirler exit

\section{Acknowledgments}

This work was supported by the ANR-07-CIS7-008-04 Grant of the French Ministry of Research. We are grateful to the CERFACS combustion team (Toulouse, France) for providing the geometry of PRECCINSTA. The IDRIS, the CNRS's national supercomputing center is acknowledged for the computational facilities. The authors warmly acknowledge the support of the 2008 Summer Program of the Center for Turbulence Research (Stanford University - NASA Ames) during which this work was initiated.

\section{References}

${ }^{1}$ U. Maas and S. Pope. Simplifying chemical kinetics: Intrinsic low-dimensional manifolds in composition space. Combust. Flame, 88:239-264, 1992.

${ }^{2}$ O. Gicquel, N. Darabiha, and D. Thévenin. Laminar premixed hydrogen / air counterflow flame simulations using flame prolongation of ildm with differential diffusion. In The Proceedings of the Twenty-Eighth Symposium (Int.) on combustion. The Combustion Institute, Pittsburgh, pages 1901-1908, 2000.

${ }^{3}$ B. Fiorina, R. Baron, O. Gicquel, D. Thévenin, S. Carpentier, and N. Darabiha. Modelling non-adiabatic partially- 


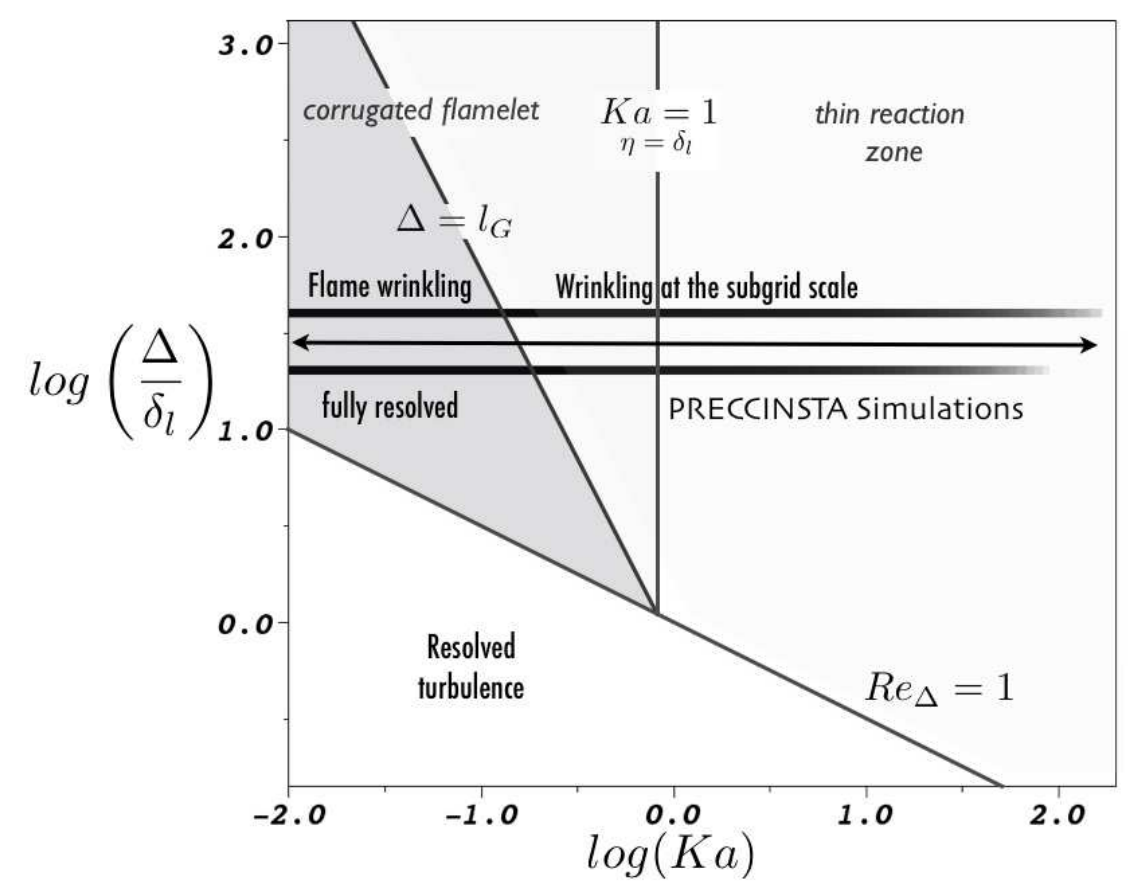

Figure 10. LES regime diagram for turbulent premixed combustion. The thick solid black lines represent the range covered by the PRECCINSTA flame simulations

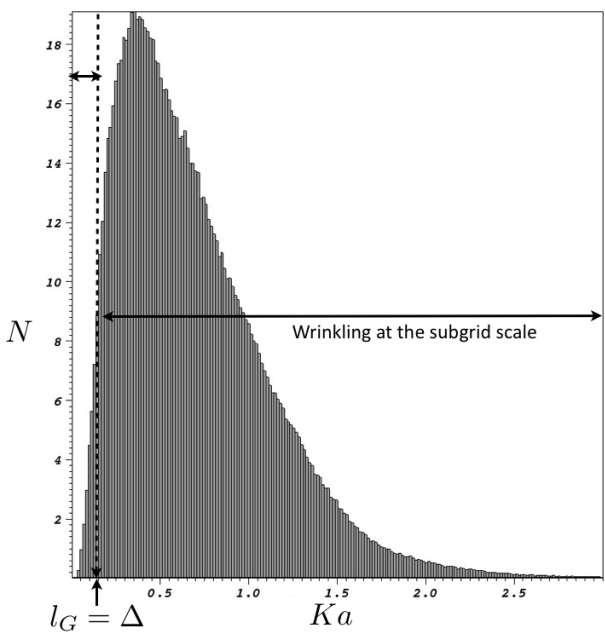

(a) $\Delta / \delta_{l}=40$

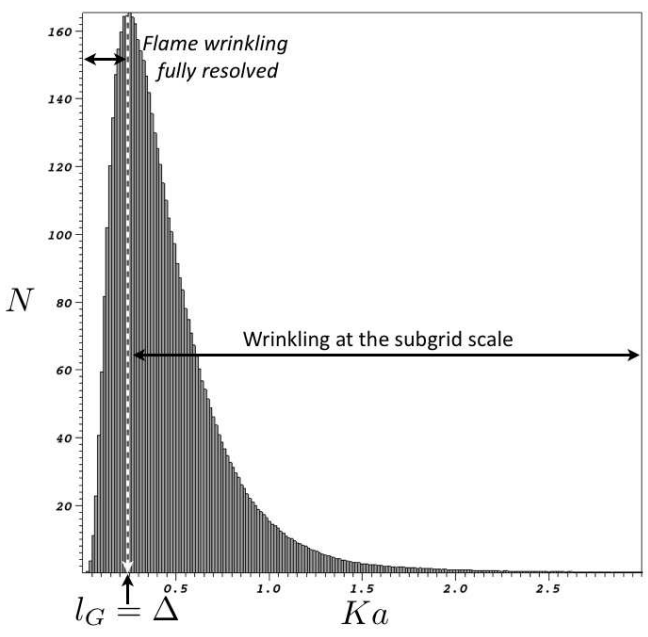

(b) $\Delta / \delta_{l}=20$

Figure 11. Node distribution versus the Karlovitz number. Only nodes located into the filtered flame front have been considered, i.e. for $0.01<\widetilde{c}<0.99$

premixed flames using flame prolongation of ildm. Combustion Theory and Modelling, 7:449-470, 2003.

${ }^{4}$ J. A. van Oijen, F. A. Lammers, and L. P. H. de Goey. Modelling of complex premixed burner systems by using flamelet-generated manifolds. Combust. Flame, 127(3):2124-2134, 2001.

${ }^{5}$ T. D. Butler and P. J. O'Rourke. A numerical method for two-dimensional unsteady reacting flows. Proceedings of the 16th Symp. (Int.) on Combustion. The Combustion Institute: Pittsburgh, Penn., pages 1503-1515, 1977.

${ }^{6}$ O. Colin, F. Ducros, D. Veynante, and T. Poinsot. A thickened flame model for large eddy simulations of turbulent premixed combustion. Physics of Fluids, 12(7):1843-1863, 2000.

${ }^{7}$ V. Moureau, B. Fiorina, and H. Pitsch. A level set formulation for premixed combustion les considering the turbulent flame structure. combust. and Flame, 156(4):801-812, 2009.

${ }^{8}$ B. Fiorina, R. Vicquelin, P. Auzillon, N. Darabiha, O. Gicquel, and D. Veynante. A filtered tabulated chemistry model for les of premixed combustion. Combustion and Flame, In Press, Corrected Proof(doi: DOI: 
10.1016/j.combustflame.2009.09.015):-, 2009.

${ }^{9}$ O. Colin, F. Ducros, D. Veynante, and T. Poinsot. A thickened flame model for large eddy simulations of turbulent premixed combustion. Physics of Fluids, 12(7):1843-1863, 2000.

${ }^{10} \mathrm{~F}$. Charlette, C. Meneveau, and D. Veynante. A power-law flame wrinkling model for les of premixed turbulent combustion part ii: dynamic formulation. Combustion and Flame, 131(1-2):181-197, 2002.

${ }^{11} \mathrm{H}$. Pitsch. A consistent level set formulation for large-eddy simulation of premixed turbulent combustion. Combustion and Flame, 143(4):587-598, 2005.

${ }^{12}$ E. R. Hawkes and R. S. Cant. A flame surface density approach to large-eddy simulation of premixed turbulent combustion. Symposium (International) on Combustion, 28(1):51-58, 2000.

${ }^{13}$ S. Richard, O. Colin, O. Vermorel, A. Benkenida, C. Angelberger, and D. Veynante. Towards large eddy simulation of combustion in spark ignition engines. Proceedings of the Combustion Institute, 31(2):3059-3066, 2007.

${ }^{14}$ R. J. Kee, J.F. Grcar, M. D. Smooke, and Miller J. A. A fortran program for modelling steady laminar one dimensional premixed flame. Tech. Rep. Sandia National Laboratories, 1992.

${ }^{15}$ W. Meier, P. Weigand, X.R. Duan, and R. Giezendanner-Thoben. Detailed characterization of the dynamics of thermoacoustic pulsations in a lean premixed swirl flame. Combustion and Flame, 150(1-2):2 - 26, 2007.

${ }^{16}$ Avbp code : www.cerfacs.fr/cfd/avbp code.php and www.cerfacs.fr/cfd/ cfdpublications.html. 2008.

${ }^{17}$ Olivier Colin and Michael Rudgyard. Development of high-order taylor-galerkin schemes for les. Journal of Computational Physics, 162(2):338-371, 82000.

${ }^{18}$ B. Fiorina, O. Gicquel, L. Vervisch, S. Carpentier, and N. Darabiha. Premixed turbulent combustion modelling using tabulated chemistry and pdf. Proc. Combust. Inst., 30:867-874, 2005.

${ }^{19}$ H. Pitsch. Large-eddy simulation of turbulent combustion. Ann. Rev. Fluid Mech., 38:453-482, 2006.

${ }^{20}$ N. Peters. Turbulent Combustion. Cambridge University Press, 2000.

${ }^{21}$ R. Vicquelin, B. Fiorina, N. Darabiha, D. Veynante, V.Moureau, and L. Vervisch. Coupling tabulated chemistry with larege-eddy simulation of turbuelnt reactive flows. Proceedings of the Summer Program, 2008. 


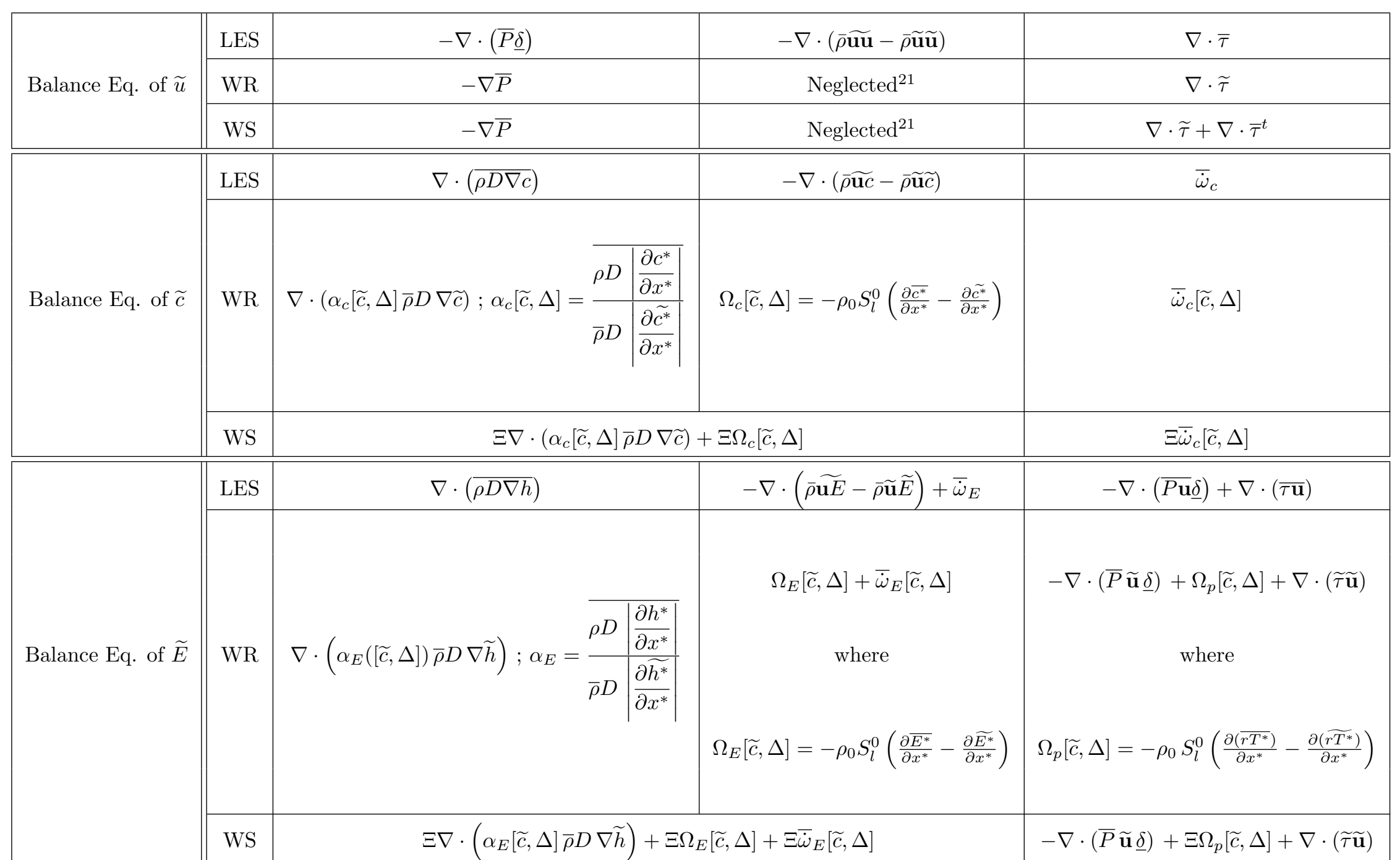

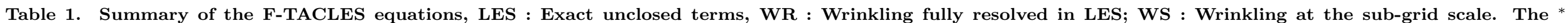
superscript denotes quantities from a 1-D unstretched laminar premixed flame 